\title{
Maternal and neonatal health care worker well-being and patient safety climate amid the COVID-19 pandemic
}

\author{
Eman Haidari $\circledast^{1} \cdot$ Elliott K. Main ${ }^{2} \cdot$ Xin Cui $\oplus^{3,4} \cdot$ Valerie Cape $^{5,6} \cdot$ Daniel S. Tawfik $^{7} \cdot$ Kathryn C. Adair ${ }^{8} \cdot$ \\ Bryan J. Sexton ${ }^{8,9} \cdot$ Jochen Profit $\mathbb{D}^{3}$
}

Received: 20 September 2020 / Revised: 11 February 2021 / Accepted: 17 February 2021 / Published online: 16 March 2021

(c) The Author(s), under exclusive licence to Springer Nature America, Inc. 2021

\begin{abstract}
Objective To assess maternal and neonatal healthcare workers (HCWs) perspectives on well-being and patient safety amid the COVID-19 pandemic.

Study design Anonymous survey of HCW well-being, burnout, and patient safety over the prior conducted in June 2020. Results were analyzed by job position and burnout status.

Result We analyzed 288 fully completed surveys. In total, $66 \%$ of respondents reported symptoms of burnout and $73 \%$ felt burnout among their co-workers had significantly increased. Workplace strategies to address HCW well-being were judged by $34 \%$ as sufficient. HCWs who were "burned out" reported significantly worse well-being and patient safety attributes. Compared to physicians, nurses reported higher rates of unprofessional behavior (37\% vs. 14\%, $p=0.027)$ and difficulty focusing on work (59\% vs. $36 \%, p=0.013$ ).

Conclusion Three months into the COVID-19 pandemic, HCW well-being was substantially compromised, with negative ramifications for patient safety.
\end{abstract}

\section{Introduction}

Maternity providers attend approximately 3.8 million births annually, with over $10 \%$ of newborns requiring care in a neonatal intensive care unit (NICU) [1-3]. Care for birthing persons and their sick infants is highly rewarding but also physically and emotionally taxing, with a quarter to half of health care workers (HCWs) reporting severe burnout, poor work-life integration, depression, or other manifestations of impaired well-being [4, 5]. Human factors in healthcare, such as HCW burnout, safety culture, and teamwork have been shown to associate strongly with quality of care delivery $[6,7]$. External influences, such as the current COVID-19 pandemic, may influence care context with potentially negative consequences for provider well-being, safety and quality of care, and outcomes of birthing persons and critically ill infants.
Jochen Profit

profit@stanford.edu

1 Division of Neonatology, Department of Pediatrics, Stanford University School of Medicine, Stanford, CA, USA

2 Medical Director of California Maternal Quality Care Collaborative, Division of Maternal Fetal Medicine, Department of Obstetrics and Gynecology, Stanford University School of Medicine, Stanford, CA, USA

3 Perinatal Epidemiology and Health Outcomes Research Unit, Division of Neonatology, Department of Pediatrics, Stanford University School of Medicine, Stanford, CA, USA

4 California Perinatal Quality Care Collaborative, Stanford, CA, USA
5 Division of Neonatology, Department of Pediatrics, Stanford University School of Medicine, Stanford, CA, USA

6 California Maternal Quality Care Collaborative, Stanford CA, USA

7 Division of Pediatric Critical Care Medicine, Department of Pediatrics, Stanford University School of Medicine, Stanford, CA, USA

8 Duke Center for Healthcare Safety and Quality, Duke University Health System, Durham, NC, USA

9 Department of Psychiatry, Duke University School of Medicine, Duke University Health System, Durham, NC, USA 
HCW burnout is a work-related, reversible syndrome primarily consisting of emotional exhaustion, decreased personal accomplishment, and/or depersonalization [8], inversely related to well-being and a culture of patient safety $[5,9]$. Burnout has been linked to detrimental effects on HCWs, organizations, and patients. For example, burned out HCWs experience higher rates of depression, alcoholism, and suicidal ideation, as well as poorer relationships with family, co-workers and patients [10-14]. Organizations suffer from increased staff turnover, decreased productivity, increased lawsuits, and worse safety culture ratings $[5,9,15,16]$. In turn, these factors associate with lower patient satisfaction, increased medical errors, and poor patient outcomes such as increased health-care associated infection rates and mortality [4, 6, 17-19]. Obstetrics and Neonatology are care settings that are particularly vulnerable to burnout $[5,20]$, in part due to the high-stakes, technical environment, and need to continuously deliver empathic care in a time-sensitive highpressure environment $[4,21]$.

The COVID-19 pandemic may further exacerbate HCW burnout and impair well-being [22], given the threat of the pandemic to human life, including concern for infections in patients, loved ones, or oneself [23]; rapidly changing information requiring frequent adjustments to care delivery systems; lack of adequate care resources including personal protective equipment and limited testing capabilities; and changes to home life responsibilities such as child care, distance learning, and restricted social interactions. However, whether the pandemic affects HCW well-being, and in which direction, has been controversial. In a perspective, Hartzband and Groopman suggested that emotional exhaustion (the most salient component of burnout for HCW) may actually decrease during the pandemic due to increased autonomy and professional recognition, and thus, perhaps HCWs would find renewed meaning in their profession [24]. Other studies however, have found increased distress due to COVID, raising concern regarding increasing burnout [22, 23, 25-28]. Acquisition of empirical data on maternal and neonatal HCW perceptions of emotional exhaustion and patient safety culture is critical, because adverse findings would be concerning for $\mathrm{HCW}$, as well as for patients. This would be significant because it would reveal an actionable pathway to reduce collateral damage from COVID-19. The objective of this study was to assess these domains during a relatively early stage of the pandemic.

\section{Methods}

\section{Design and participants}

Cross-sectional survey study of obstetric and neonatal HCWs. We invited a convenience sample of HCWs who had registered for the California Maternal/Perinatal Quality Care Collaborative (CM/PQCC) "Mental Health Considerations during the COVID-19 Pandemic" webinar to complete an anonymous electronic survey via Stanford's Qualtrics ${ }^{\mathrm{XM}}$ platform. The webinar was one of a series on COVID-19 held 4/29/2020. There were 1725 registrants and 673 attended. Participation was targeted to California HCWs, but was also open to out-of-state participants. The 3-5 min HCW survey was administered between 5/26/20 and $6 / 8 / 20$ to webinar registrants after receipt of IRB approval. Potential respondents received two reminder emails during that period. Participation and completion of individual items was voluntary, and responses were anonymous. At the time of the survey, California experienced a steady, moderate number of cases ( 2500/day) and deaths ( $60 /$ day), predominantly in the southern regions of the state. Maternity units were more directly affected than NICUs, with higher case loads in hospitals serving vulnerable populations.

\section{Measures}

The survey consisted of 13 well-being and patient safety items, along with demographic information. All items asked participants to relate questions to their experience over the preceding month, and used a 5-point Likert scale ranging from disagree strongly to agree strongly. Eight items were novel and based on literature review of the suggested effect of COVID-19 on mental health and patient safety culture. These items were developed by JP, revised among the authors, pilot tested among five colleagues, which included content and methods experts in relevant clinical and wellness areas, and further revised for content and clarity as needed. They covered domains of patient safety, teamwork, work-life integration, and well-being in the context of COVID-19. Respondents were asked to consider the following items during the month prior to the survey: "It has been difficult to fulfill home and work responsibilities"; "Unprofessional behavior in my work setting has affected my well-being"; "Support from colleagues has lifted me up"; "I have been hopeful about the future"; "Existing strategies to improve staff well-being in my work setting were sufficient"; "Burnout among my co-workers has significantly increased"; "My co-workers have had difficulty focusing on their work"; and "Medical errors have increased in my work setting". Items include a mix of assessments of respondents and their colleagues to reduce participant's social desirability bias in questions about patient safety.

We evaluated burnout with a widely used [9, 19, 29-32] 5-item emotional exhaustion scale shown to have excellent psychometric properties [19, 29, 32-34], external validity [9, 31, 33], and responsiveness to interventions [19, 32, 34]. To limit response burden, particularly during the pandemic, 
we elected to forgo assessment, of depersonalization and personal accomplishment to reduce survey burden. According to a psychometric meta-analysis, of the three sub-scales of burnout (emotional exhaustion, depersonalization, and personal accomplishment), emotional exhaustion consistently produces the largest and most consistent coefficient alpha estimates [35]. Self-reported demographics included respondent role, years in current position, job area, ACOG [36] and AAP level of care [37], location (state/ country), and sex. For job position analyses, we pooled respondents into "physician" (physician, NP/PA), "nurse" (nurse, CNS/educator, CNM, nurse manager), and "other" (hospital manager/administrator, social worker, behavioral health, academia, quality staff/provider, and other) groups due to the small number of respondents from some of the categories $[5,38]$.

\section{Analysis}

Respondents from outside the United States were excluded from analyses. Frequencies and proportions were used to describe survey responses and respondent demographics. For ease of interpretability, we defined a "percent concerning" measure to indicate the proportion of respondents reporting undesirable results. Questions are transformed from the 5-point Likert scale to a 100-point scale. We used the established threshold of 50 or higher, averaged across scale items [9, 19, 29, 33, 39], to reflect "not disagreeing," with emotional exhaustion items. For each of the novel items we calculated percent agreement by combining agree slightly with agree strongly as a fraction of all respondents. We stratified analyses by maternal vs. neonatal HCWs, as well as by neonatal or maternal level of care. We used chi square tests and ANOVA to evaluate response differences across subgroups. All statistical analyses were performed using SAS version 9.4. For perspective, we compared emotional exhaustion in this neonatal and maternal HCW sample with prior samples of $2073 \mathrm{HCWs}$ in 44 California NICUs in 2011 [5] and 10,627 HCWs from 829 work settings in 31 hospitals in Michigan in 2016 [33]. This study was approved by the Stanford University Institutional Review Board (\#56642).

\section{Results}

After excluding non-United States HCWs, our sample included 1725 eligible HCWs who registered for the webinar, of which 673 participated in the webinar. Of these, 288 fully completed the survey sent to them 1 month after the webinar (reflecting up to $17 \%$ of registrants or $43 \%$ of webinar attendees). A majority of the respondents were nurses and female, with nearly half reporting more than 5 years of work experience in their current position. Maternity care respondents mostly worked in ACOG level II and III (highest level of care) facilities whereas most neonatal respondents worked in AAP level III, with about $1 / 8$ distributed to each of the other levels of care. About one third of respondents were from California (Table 1).

Figure 1 shows item level responses. Dichotomized responses (agree slightly and agree strongly) reveal that $12 \%$ reported increased medical errors, $33 \%$ reported more unprofessional behavior, $58 \%$ reported co-workers having difficulty focusing on work, and $59 \%$ reported difficulty meeting home and work responsibilities. Only 34\% of respondent reported sufficient institutional strategies to meet these well-being challenges. Notably, $70 \%$ of respondents were hopeful about the future and $83 \%$ felt lifted up by their colleagues, and simultaneously $73 \%$ perceived an increase in burnout among their co-workers. In total, $66 \%$ of respondents reported symptoms consistent with emotional exhaustion.

Compared to physicians, nurses reported higher percentages concerning for unprofessional behavior (37\% vs. $15 \%, p=0.036)$ and difficulty focusing on work (60\% vs. $38 \%, p=0.024)$. A perceived increased in medical errors was reported by $14 \%$ of nurses, compared to $6 \%$ of physicians $(p=0.337)$. Figure 2 shows consistently worse ratings across all items among respondents with burnout (emotional exhaustion) compared to those without burnout. Responses were similar across specialties and levels of care, the only significant difference being less sufficient available institutional resources for well-being among higher ACOG defined levels of maternity care (L I $51 \%$, L II 38\%, L III $30 \%, p=0.039)$.

Figure 3 shows the current survey results in context to prior assessments of burnout among 2073 HCWs from California in 2011 and 10,627 HCWs from Michigan in 2016. The current observed prevalence of burnout of $66 \%$ is consistent with work settings in the highest quintile from prior assessments.

\section{Discussion}

This survey provides a summary of HCW well-being and care culture among a sample of neonatal and maternity care providers during the COVID-19 pandemic. In our sample, $73 \%$ of respondents reported increased coworker burnout and $66 \%$ reported personal symptoms of emotional exhaustion in the month prior to the survey, which correlated with the early phase of the COVID-19 pandemic response in the United States. Most respondents also expressed difficulty balancing home and work responsibilities, and nearly half felt that existing institutional strategies were unable to cushion these effects. As a group, 
Table 1 Demographics characteristics of the study population.

\begin{tabular}{|c|c|c|}
\hline & $N$ & $\%$ \\
\hline Total & 288 & 100.0 \\
\hline \multicolumn{3}{|l|}{ Gender } \\
\hline Male & 10 & 3.5 \\
\hline Female & 277 & 96.2 \\
\hline Prefer not to answer & 1 & 0.3 \\
\hline \multicolumn{3}{|l|}{ Role of work $^{\mathrm{a}}$} \\
\hline MD & 34 & 11.8 \\
\hline $\mathrm{RN}$ & 169 & 58.7 \\
\hline Other & 85 & 29.5 \\
\hline \multicolumn{3}{|c|}{ Years working in the current position } \\
\hline Less than 1 year & 32 & 11.1 \\
\hline $1-5$ years & 112 & 38.9 \\
\hline $6-10$ years & 36 & 12.5 \\
\hline Greater than 10 years & 106 & 36.8 \\
\hline \multicolumn{3}{|c|}{ Specialty $\left(\right.$ select all apply) ${ }^{\mathrm{b}}$} \\
\hline $\mathrm{OB}$ & 209 & 72.6 \\
\hline Neo & 134 & 46.5 \\
\hline Other & 93 & 32.3 \\
\hline \multicolumn{3}{|c|}{ ACOG level of maternal care ${ }^{\mathrm{c}}$} \\
\hline Level I & 35 & 12.2 \\
\hline Level II & 105 & 36.5 \\
\hline Level III & 91 & 31.6 \\
\hline N/A & 45 & 15.6 \\
\hline Do not know & 9 & 3.1 \\
\hline \multicolumn{3}{|c|}{ AAP level of neonatal care ${ }^{\mathrm{d}}$} \\
\hline Level I & 41 & 14.2 \\
\hline Level II & 46 & 16.0 \\
\hline Level III & 103 & 35.8 \\
\hline Level IV & 41 & 14.2 \\
\hline N/A & 51 & 17.7 \\
\hline Do not know & 4 & 1.4 \\
\hline \multicolumn{3}{|l|}{ Current residential state } \\
\hline California & 104 & 36.1 \\
\hline Non-California ${ }^{\mathrm{e}}$ & 184 & 63.9 \\
\hline
\end{tabular}

${ }^{\mathrm{a}} \mathrm{MD}$ includes: physician, NP/PA; RN includes: nurse, CNS/Educator, $\mathrm{CNM}$, nurse manager; Other includes: Hospital manager/Administrator, social worker, behavioral health, Academia, Quality staff/ Provider, and other.

${ }^{b} \mathrm{OB}$ includes: labor and delivery, antepartum, postpartum; Neo includes: newborn nurse, NICU; other includes Emergency Department, Medical/Surgical, operating room, ambulatory care, Patient/ Family support services, and other.

${ }^{c}$ Level I: basic care of low-risk to moderate-risk pregnancies; Level II: specialty care of low-risk, moderate-risk, and high-risk pregnancies; Level III: subspecialty care for more complex conditions and complications.

${ }^{\mathrm{d}}$ Level I: Well newborn nursery; Level II: special care nursery; Level III: neonatal intensive-care unit (NICU); Level IV: regional neonatal intensive-care unit (regional NICU).

${ }^{\mathrm{e}}$ Non-California includes Washington, DC and 40 states in the United States. States without respondents included are Alaska, Delaware, Hawaii, Maine, North Dakota, South Carolina, South Dakota, Vermont, and Wyoming.

Data may not add to $100 \%$ due to low percentage of missing across response categories (all $<1.1 \%$ ). respondents that experienced burnout rated all other wellbeing and safety items significantly worse.

For purposes of context and anchoring, we relayed current results within prior samples of neonatal and other hospital-level health care workers. Although these comparisons should be treated with caution, given the different populations and sampling methods, it is notable that burnout prevalence was higher than in any of 44 NICUs surveyed in 2011, and in the highest quintile for 829 work settings in 31 Michigan hospitals in 2016 [33]. The timing of this survey during the third month of the US response to the COVID-19 pandemic suggests that pandemic-related disruptions to work life and personal life were likely key contributors to the increased burnout prevalence. However, it may still be too early to fully understand the effect of COVID-19 on burnout, because we saw higher depression rates at 1-year follow-up of HCWs with high-risk exposure during the SARS outbreak [40].

Several mechanisms may explain an increase in burnout described in our study, viewed in light of the job demandsresources model of burnout [41, 42]. Although maternity and neonatal providers are less likely to directly care for COVID-19 patients relative to other specialties, job demands may nevertheless be increased. Demands may be increased due to frequently changing infection control processes, social distancing protocols at work, and additional testing and isolation of providers and patients [43]. Job resources may also be reduced, with support personnel delegated to additional roles or moved remotely, and staffing shortages secondary to infection exposures or budget shortfalls. Maternity care providers have been more exposed to SARS-CoV-2 positive patients ( $10 \%$ of pregnant women), as positivity has been rare in newborns [44]. Yet, direct exposure to a contagious patient is relatively uncommon compared to emergency room or adult intensive care units. Nevertheless, we find high levels of disruption to $\mathrm{HCW}$ well-being. Direct exposure risk may thus not correlate well with HCW well-being. It is possible that stressors other than direct exposures can exert a similar emotional toll. For example, maternal and neonatal HCWs may experience substantial distress when separating mothers and families from their newborn [45]. Such distress is warranted. Fears of separation from the newborn and support persons during the pandemic appears to be fueling a rise in home and birth center births [46].

At the personal level, self-isolation, disrupted societal resources, limited childcare, and family concerns may all contribute to depleted physical and emotional resources, resulting in emotional exhaustion. Although an initial outpouring of support for HCWs during the acute phase of the pandemic response may have counteracted many of these 


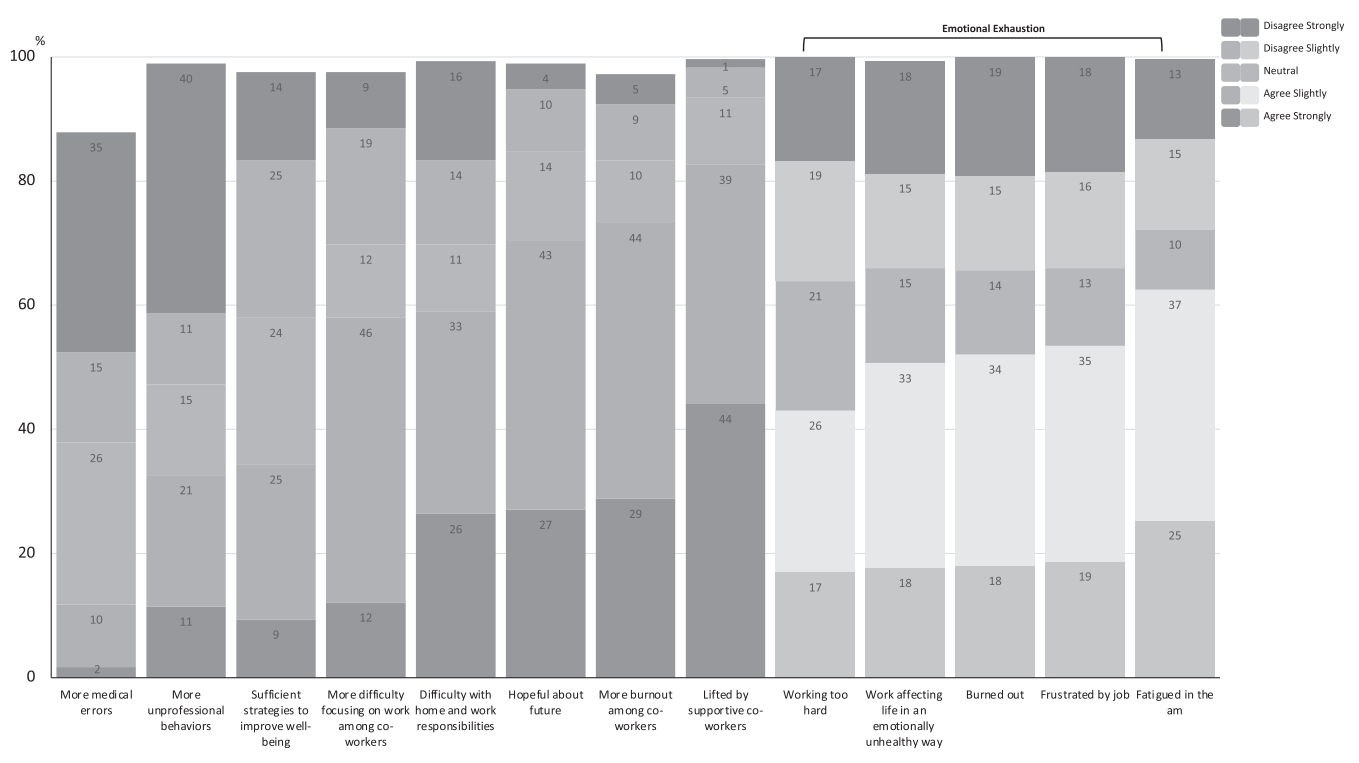

Fig. 1 Item level and scale level survey responses. Eight novel items to the left, five emotional exhaustion scale items to the right. Data were sorted by increasing \% agreement (including Agree Strongly and Agree Slightly).

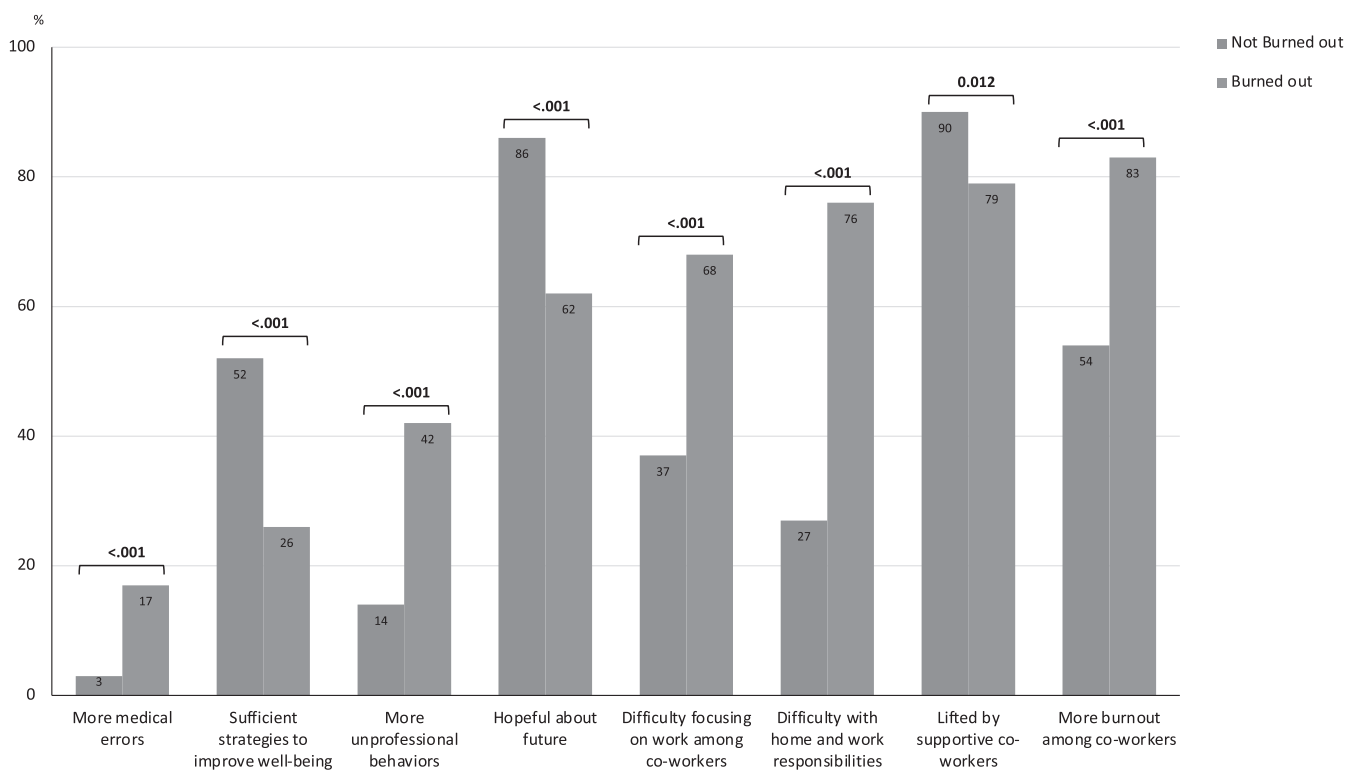

Fig. 2 Survey results stratified by respondent burnout status. Data were sorted by increasing \% agreement among respondents with burnout (including Agree Strongly, Agree Slightly).

challenges, and even contributed to improved professional satisfaction and meaning in work [24], our findings suggest that any such effects may have been transient.

Relative to other published studies, the $66 \%$ reporting emotional exhaustion here is quite high. Prior research has shown that care quality and quality improvement efforts suffer as emotional exhaustion rates start to exceed $40 \%$ $[5,34]$. Our results suggest that the emotional upheaval of the global health crisis may have impacted healthcare worker well-being sufficiently to impact patient safety. The precise impact of the pandemic on these relations is not known, and to date, no studies of healthcare workers have addressed this question directly. Nevertheless, unpacking the impact of COVID-19 on HCW well-being, care quality, and quality improvement efforts will take several years and extensive efforts to address.

Only a third of respondents felt that existing strategies to improve staff well-being in the workplace were sufficient, indicating a dire need for effective strategies to address burnout during such times of upheaval. Yet, promoting HCW well-being should be of utmost priority to institutions as burnout is directly correlated with malpractice lawsuits, 


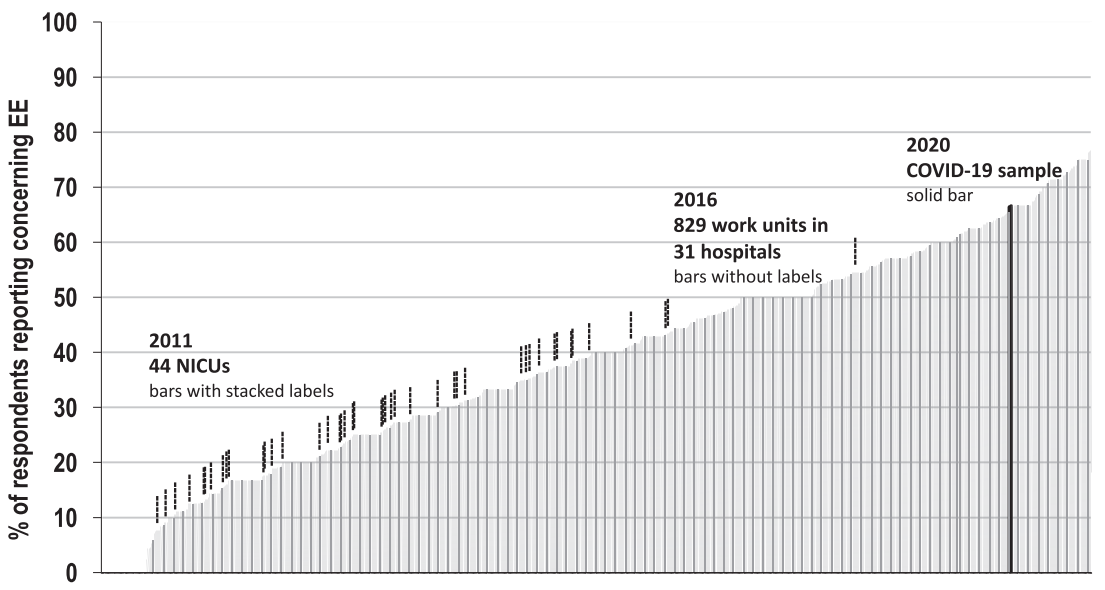

Fig. 3 Comparison of burnout (emotional exhaustion) in current sample with prior NICU and adult samples. Each bar shows \% respondents reporting moderate to severe burnout in either the current sample, a NICU, or a hospital work setting. COVID-19 June, 2020 sample (solid bar; mean \% reporting emotional exhaustion was 66\%). Sample from 44 California NICUs in 2011 (stacked bar labeled;

costly staff turnover, productivity, relationships among staff, patients safety, clinical outcomes, and patient satisfaction [5, 19, 28, 47-52]. Among our sample, maternity providers in centers with higher ACOG levels of maternity care felt that strategies to combat burnout were less sufficient. This suggests that institutions with higher levels of care may require relatively more aggressive strategies to support HCW well-being [22]. Indeed, over the past years, academic healthcare organizations have led the industry and established wellness offices tasked with identifying and employing effective strategies to combat burnout and improve HCW well-being [53]. However, our survey suggests that these efforts still have challenges in meeting frontline provider need. Current offerings may either fail to reach frontline providers or have not yet penetrated work culture in a way sufficient to alleviate chronic stress and suffering. Approaches to promote $\mathrm{HCW}$ well-being and address burnout are still evolving. Both structural and individual-focused interventions have been effective in combating burnout among HCWs [54]. Structural interventions in theory may carry benefits of better penetrance to the HCWs within a system and of longer-lasting effects. Conversely, individual-focused interventions may carry the benefits of rapid deployment and adoption with minimal resource expenditure, and many are conducive to remote engagement using smartphones or other technology-features that may be particularly beneficial during the highly dynamic and taxing environment of a pandemic response. We have found that simple, bite-sized strategies are favorably received by HCWs, and have developed a family of these interventions for healthcare workers (www.hsq. dukehealth.org/tools) [32]. mean \% reporting emotional exhaustion was $26 \%$ ) used a 4-item scale to which we subsequently added an additional item for improved construct validity. Unit level correlation between scales exceeds 0.98 . Sample from 829 work settings in 31 hospitals in Michigan in 2016 (no label; mean \% reporting emotional exhaustion was $40 \%$ ) responded to the 5 -item scale.

While many respondents noted a sense of support from their colleagues and expressed hope about the future, we found concerning negative consequences for patient safety culture, with respondents reporting difficulty focusing on work, unprofessional behavior, and a small (12\%) perceived rise in medical errors. Obstetric and NICU care requires great attention to detail. Nurses execute more than 100 discrete work processes per shift and inattention can lead to imperfections with potentially severe adverse consequences [55]. Increases in shift length during the pandemic may further exacerbate errors [56]. Furthermore, a rise in errors (real or perceived) could be associated with the increase in burnout due to added stress from COVID-19, as higher burnout rates are well known to be correlated with an increase in medical errors $[5,18,47,57]$. However, this is speculative and beyond the direct findings of this survey. In addition, the directionality of the relationship between burnout and errors in this survey is unknown, although there is good reason to expect bidirectionality $[58,59]$.

Of note, nurses were more likely than physicians to agree that their well-being was impacted by unprofessional behavior in the work setting, and that co-workers had difficulty focusing on their work. This is consistent with previous literature about differing perceptions of teamwork, in which nurses reported more impact and concern with unprofessional behavior than their physician counterparts [60]. Nearly $60 \%$ of respondents reported difficulty fulfilling both home and work responsibilities. Although one might expect this to be higher given the concurrent closure of schools/ daycares, demographic data did not include information on spouses/partners or dependents at home. 
This study should be interpreted in light of its design. Our analyses describe associations, not causal relationships and should be interpreted accordingly. We didn't correct for multiple testing. Thus, results should be viewed as hypothesis generating, subject to Type 1 error. Survey respondents consisted of a self-selected sample of HCWs available to complete a survey a month following a webinar they may or may not have attended. It is also possible that HCWs directly experiencing an increase in high acuity COVID-19 patients, or other extreme time demands were not included. We would expect such an omission to bias results toward the null, meaning the prevalence of HCW burnout observed in our study may be an underestimate. Conversely, it is possible that nonrespondents don't perceive the current pandemic to influence either HCW well-being or patient safety, biasing our results toward a more extreme finding. While the direction of non-response bias is unknown, our study's response rate compares favorably with other published literature [61]. For confidentiality and brevity, respondents were not asked to identify their specific healthcare organizations or describe their personal resources and needs, preventing comparisons among healthcare settings and family situations. For parsimony, we used emotional exhaustion as a surrogate for burnout, but we may have not fully captured other manifestations of burnout such as depersonalization or reduced personal accomplishment. The novel items by definition have no baseline comparisons. Although we asked respondents to assess these in comparison to the prior month, attribution of changes to the pandemic requires caution. We also lacked information on regional differences or whether respondents were working in their usual work settings. Finally, although this study represents a snapshot of well-being and patient safety perceptions of maternal and neonatal HCWs, generalizability is strengthened by the variety of care settings and respondents from 40 states and the District of Columbia, which together account for more than $96 \%$ of the population.

In conclusion, HCW well being appears to have deteriorated during the pandemic with $73 \%$ of respondents endorsed an increase in co-worker burnout and 66\% personal emotional exhaustion. Moreover, only a third reported sufficient institutional strategies to meet these well-being challenges. Those who reported symptoms of burnout were particularly likely to also endorse negative work setting impact. With the COVID-19 pandemic ongoing, burnout and its ramifications for clinical care will likely increase unless addressed. Interestingly, despite the high burnout rates, the majority of respondents were hopeful (70\%). This could be due to the fact that COVID-19 may bring meaning to the work, and thus it may play a role in decreasing emotional exhaustion in the short term [22, 24], but longer term effects are still unknown. Thus, action should be taken now as burnout and its downstream effects are likely to continue to increase if not addressed.

Funding This work was supported by the Eunice Kennedy Shriver National Institute of Child Health and Human Development [R01 HD084679-01, Co-PI: Sexton and Profit] and by National Institutes of Health Clinical Investigator Award [K08 HS27837-01, PI: Daniel Tawfik].

Author contributions Dr. Profit conceptualized the study and reviewed and revised the initial manuscript; Dr. Haidari drafted the initial manuscript; Dr. Cui conducted data analysis; and all authors provided interpretation of data, reviewed and revised the manuscript critically for important intellectual content, and approved the final manuscript as submitted.

\section{Compliance with ethical standards}

Conflict of interest The authors declare no competing interests.

Publisher's note Springer Nature remains neutral with regard to jurisdictional claims in published maps and institutional affiliations.

\section{References}

1. Special Care Nursery Admissions. March of Dimes. 2011. https://www.marchofdimes.org/peristats/pdfdocs/nicu_summary_ final.pdf.

2. Edwards EM, Horbar JD. Variation in use by NICU types in the United States. Pediatrics. 2018;142:e20180457.

3. Martin JA, Hamilton BE, Osterman MJK, Driscoll AK. Births: final data for 2018. Hyattsville: National Center for Health Statistics; 2019.

4. Tawfik DS, Profit J. Provider burnout: implications for our perinatal patients. Semin Perinatol. 2020;44:151243.

5. Profit J, Sharek PJ, Amspoker AB, Kowalkowski MA, Nisbet CC, Thomas EJ, et al. Burnout in the NICU setting and its relation to safety culture. BMJ Qual Saf. 2014;23:806-13.

6. Tawfik DS, Scheid A, Profit J, Shanafelt T, Trockel M, Adair KC, et al. Evidence relating health care provider burnout and quality of care: a systematic review and meta-analysis. Ann Intern Med. 2019;171:555-67.

7. Profit J, Sharek PJ, Cui X, Nisbet CC, Thomas EJ, Tawfik DS, et al. The correlation between neonatal intensive care unit safety culture and quality of care. J Patient Saf. 2020;16:e310-6.

8. Maslach C, Schaufeli WB, Leiter MP. Job burnout. Annu Rev Psychol. 2001;52:397-422.

9. Schwartz SP, Adair KC, Bae J, Rehder KJ, Shanafelt TD, Profit J, et al. Work-life balance behaviours cluster in work settings and relate to burnout and safety culture: a cross-sectional survey analysis. BMJ Qual Saf. 2019;28:142-50.

10. Center C, Davis M, Detre T, Ford DE, Hansbrough W, Hendin H, et al. Confronting depression and suicide in physicians: a consensus statement. J Am Med Assoc. 2003;289:3161-6.

11. Oreskovich MR, Shanafelt T, Dyrbye LN, Tan L, Sotile W, Satele $\mathrm{D}$, et al. The prevalence of substance use disorders in American physicians. Am J Addict. 2015;24:30-8.

12. Shanafelt TD, Balch CM, Dyrbye L, Bechamps G, Russell T, Satele D, et al. Special report: suicidal ideation among American surgeons. Arch Surg. 2011;146:54-62.

13. Dyrbye LN, Shanafelt TD, Balch CM, Satele D, Sloan J, Freischlag J. Relationship between work-home conflicts and burnout 
among American surgeons: a comparison by sex. Arch Surg. 2011;146:211-7.

14. Dyrbye LN, West CP, Satele D, Sloan JA, Shanafelt TD. Work/ Home conflict and burnout among academic internal medicine physicians. Arch Intern Med. 2011;171:1207-9.

15. Han S, Shanafelt TD, Sinsky CA, Awad KM, Dyrbye LN, Fiscus LC, et al. Estimating the attributable cost of physician burnout in the United States. Ann Intern Med. 2019;170:784-90.

16. Shanafelt T, Goh J, Sinsky C. The business case for investing in physician well-being. JAMA Intern Med. 2017;177:1826-32.

17. Tawfik DS, Sexton JB, Kan P, Sharek PJ, Nisbet CC, Rigdon J, et al. Burnout in the neonatal intensive care unit and its relation to healthcare-associated infections. J Perinatol. 2017;37:315-20.

18. Tawfik DS, Profit J, Morgenthaler TI, Satele DV, Sinsky CA, Dyrbye LN, et al. Physician burnout, well-being, and work unit safety grades in relationship to reported medical errors. Mayo Clin Proc. 2018;93:1571-80.

19. Sexton JB, Adair KC. Forty-five good things: a prospective pilot study of the three good things well-being intervention in the USA for healthcare worker emotional exhaustion, depression, work-life balance and happiness. BMJ Open. 2019;9:e022695.

20. Weintraub AS, Geithner EM, Stroustrup A, Waldman ED. Compassion fatigue, burnout and compassion satisfaction in neonatologists in the US. J Perinatol. 2016;36:1021-6.

21. Prentice TM, Gillam L, Davis PG, Janvier A. The use and misuse of moral distress in neonatology. Semin Fetal Neonatal Med. 2018;23:39-43.

22. Shechter A, Diaz F, Moise N, Anstey DE, Ye S, Agarwal S, et al. Psychological distress, coping behaviors, and preferences for support among New York healthcare workers during the COVID19 pandemic. Gen Hosp Psychiatry. 2020;66:1-8.

23. Fihn SD. COVID-19-back to the future. JAMA Intern Med. 2020;180:1149-50.

24. Hartzband P, Groopman J. Physician burnout, interrupted. N Engl J Med. 2020;382:2485-7.

25. Ranney ML, Griffeth V, Jha AK. Critical supply shortages-the need for ventilators and personal protective equipment during the covid-19 pandemic. N Engl J Med. 2020;382:e41.

26. Krystal JH, McNeil RL Jr. Responding to the hidden pandemic for healthcare workers: stress. Nat Med. 2020;26:639.

27. Du J, Dong L, Wang T, Yuan C, Fu R, Zhang L, et al. Psychological symptoms among frontline healthcare workers during COVID-19 outbreak in Wuhan. Gen Hosp Psychiatry 2020;67:144-5.

28. Chew NWS, Lee GKH, Tan BYQ, Jing M, Goh Y, Ngiam NJH, et al. A multinational, multicentre study on the psychological outcomes and associated physical symptoms amongst healthcare workers during COVID-19 outbreak. Brain, Behav Immun. 2020; 88:559-65.

29. Sexton JB, Adair KC, Leonard MW, Frankel TC, Proulx J, Watson SR, et al. Providing feedback following leadership walkrounds is associated with better patient safety culture, higher employee engagement and lower burnout. BMJ Qual Saf. 2018;27:261-70.

30. Rice HE, Lou-Meda R, Saxton AT, Johnston BE, Ramirez CC, Mendez S, et al. Building a safety culture in global health: lessons from Guatemala. BMJ Glob Health. 2018;3:e00630.

31. Sexton JB, Schwartz SP, Chadwick WA, Rehder KJ, Bae J, Bokovoy $\mathrm{J}$, et al. The associations between work-life balance behaviours, teamwork climate and safety climate: cross-sectional survey introducing the work-life climate scale, psychometric properties, benchmarking data and future directions. BMJ Qual Saf. 2017;26:632-40.

32. Adair KC, Rodriguez-Homs LG, Masoud S, Mosca PJ, Sexton JB. Gratitude at work: prospective cohort study of a web-based, single-exposure well-being Intervention for Health Care Workers. J Med Internet Res. 2020;22:e15562.

33. Adair KC, Quow K, Frankel A, Mosca PJ, Profit J, Hadley A, et al. The improvement readiness scale of the SCORE survey: a metric to assess capacity for quality improvement in healthcare. BMC Health Serv Res. 2018;18:975.

34. Adair KC, Kennedy LA, Sexton JB. 3 Good tools: positively reflecting backwards and forwards is associated with robust improvements in well-being across 3 distinct interventions. Cond Accept J Posit Psychol. 2019;15:613-22.

35. Wheeler DL, Vassar M, Worley JA, Barnes LLB. A reliability generalization meta-analysis of coefficient alpha for the Maslach Burnout Inventory. Educ Psychol Meas. 2011;71:231-44.

36. Levels of Maternal Care. Obstetric care consensus No, 9. Obstet Gynecol. 2019;134:e41-55.

37. Stark AR. Levels of neonatal care. Pediatrics. 2012;130:587-97.

38. Profit J, Etchegaray J, Petersen LA, Sexton JB, Hysong SJ, Mei $M$, et al. Neonatal intensive care unit safety culture varies widely. Arch Dis Child Fetal Neonatal Ed. 2012;97:F120-6.

39. Rehder KJ, Adair KC, Hadley A, McKittrick K, Frankel A, Leonard $\mathrm{M}$, et al. Associations between a new disruptive behaviors scale and teamwork, patient safety, work-life balance, burnout, and depression. Jt Comm J Qual Patient Saf 2019;46:18-26.

40. McAlonan GM, Lee AM, Cheung V, Cheung C, Tsang KW, Sham PC, et al. Immediate and sustained psychological impact of an emerging infectious disease outbreak on health care workers. Can J Psychiatry. 2007;52:241-7.

41. Bakker AB, Demerouti E. Job demands-resources theory: taking stock and looking forward. J Occup Health Psychol. 2017;22: 273-85.

42. Demerouti E, Bakker AB, Nachreiner F, Schaufeli WB. The job demands-resources model of burnout. J Appl Psychol. 2001;86: 499-512.

43. Lavizzari A, Klingenberg C, Profit J, Zupancic JAF, Davis AS, Mosca F, et al. International comparison of guidelines for managing neonates at the early phase of the SARS-CoV-2 pandemic. Pediatr Res. 2020;15:1-12.

44. Allotey J, Stallings E, Bonet M, Yap M, Chatterjee S, Kew T, et al. Clinical manifestations, risk factors, and maternal and perinatal outcomes of coronavirus disease 2019 in pregnancy: living systematic review and meta-analysis. BMJ. 2020;370:m3320.

45. Stuebe A. Should infants be separated from mothers with COVID19? First, do no harm. Breastfeed Med. 2020;15:351-2.

46. Profit J, Edmonds BT, Shah N, Cheyney M. The COVID-19 pandemic as a catalyst for a more integrated maternity care system. Am J Public Health 2020;110:1663-5.

47. Shanafelt TD, Balch CM, Bechamps G, Russell T, Dyrbye L, Satele D, et al. Burnout and medical errors among American surgeons. Ann Surg. 2010;251:995-1000.

48. van der Heijden F, Dillingh G, Bakker A, Prins J. Suicidal thoughts among medical residents with burnout. Arch Suicide Res. 2008;12:344-6.

49. Dewa CS, Jacobs P, Thanh NX, Loong D. An estimate of the cost of burnout on early retirement and reduction in clinical hours of practicing physicians in Canada. BMC Health Serv Res. 2014; $14: 254$.

50. Dewa CS, Loong D, Bonato S, Thanh NX, Jacobs P. How does burnout affect physician productivity? A systematic literature review. BMC Health Serv Res. 2014;14:325.

51. Welp A, Meier LL, Manser T. Emotional exhaustion and workload predict clinician-rated and objective patient safety. Front Psychol. 2014;5:1573.

52. Mommersteeg PM, Heijnen CJ, Kavelaars A, van Doornen LJ. Immune and endocrine function in burnout syndrome. Psychosom Med. 2006;68:879-86. 
53. Shanafelt TD, Lightner DJ, Conley CR, Petrou SP, Richardson JW, Schroeder PJ, et al. An organization model to assist individual physicians, scientists, and senior health care administrators with personal and professional needs. Mayo Clin Proc. 2017;92:1688-96.

54. West CP, Dyrbye LN, Erwin PJ, Shanafelt TD. Interventions to prevent and reduce physician burnout: a systematic review and meta-analysis. Lancet. 2016;388:2272-81.

55. Rogowski JA, Staiger DO, Patrick TE, Horbar JD, Kenny MJ, Lake ET. Nurse staffing in neonatal intensive care units in the United States. Res Nurs health. 2015;38:333-41.

56. Stimpfel AW, Lake ET, Barton S, Gorman KC, Aiken LH. How differing shift lengths relate to quality outcomes in pediatrics. $\mathbf{J}$ Nurs Adm. 2013;43:95-100.

57. Lake ET, Hallowell SG, Kutney-Lee A, Hatfield LA, Del Guidice M, Boxer BA, et al. Higher quality of care and patient safety associated with better NICU work environments. J Nurs care Qual. 2016;31:24-32.

58. Wu AW. Medical error: the second victim. West J Med. 2000; 172:358-9.

59. West CP, Huschka MM, Novotny PJ, Sloan JA, Kolars JC, Habermann TM, et al. Association of perceived medical errors with resident distress and empathy: a prospective longitudinal study. JAMA. 2006;296:1071-8.

60. Profit J, Sharek PJ, Kan P, Rigdon J, Desai M, Nisbet CC, et al. Teamwork in the NICU setting and its association with health care-associated infections in very low-birth-weight infants. Am J Perinatol. 2017;34:1032-40.

61. Shanafelt TD, West CP, Sinsky C, Trockel M, Tutty M, Satele $\mathrm{DV}$, et al. Changes in burnout and satisfaction with work-life integration in physicians and the general US working population between 2011 and 2017. Mayo Clin Proc. 2019;94:1681-94. 